\title{
Prostate-Specific Membrane Antigen Ligands for Imaging and Therapy
}

\author{
Matthias Eiber ${ }^{1,2}$, Wolfgang P. Fendler ${ }^{1,3}$, Steven P. Rowe ${ }^{4}$, Jeremie Calais ${ }^{1}$, Michael S. Hofman ${ }^{5}$, Tobias Maurer ${ }^{6}$, \\ Sarah M. Schwarzenboeck ${ }^{7}$, Clemens Kratowchil ${ }^{8}$, Ken Herrmann ${ }^{9}$, and Frederik L. Giesel ${ }^{8}$ \\ ${ }^{1}$ Department of Molecular and Medical Pharmacology, David Geffen School of Medicine, UCLA, Los Angeles, California; \\ ${ }^{2}$ Department of Nuclear Medicine, Klinikum Rechts der Isar, Technical University of Munich, Munich, Germany; ${ }^{3}$ Department of \\ Nuclear Medicine, Ludwig-Maximilians-University of Munich, Munich, Germany; ${ }^{4}$ Russell H. Morgan Department of Radiology and \\ Radiological Science, School of Medicine, Johns Hopkins University, Baltimore, Maryland; ${ }^{5}$ Division of Radiation Oncology and \\ Cancer Imaging, Peter MacCallum Cancer Centre, Melbourne, Victoria, Australia; ${ }^{6}$ Department of Urology, Klinikum Rechts der \\ Isar, Technical University of Munich, Munich, Germany; ${ }^{7}$ Department of Nuclear Medicine, Rostock University Medical Centre, \\ Rostock, Germany; ${ }^{8}$ Department of Nuclear Medicine, University Hospital Heidelberg, Heidelberg, Germany; and ${ }^{9}$ Klinik für \\ Nuklearmedizin, Universitätsklinikum Essen, Essen, Germany
}

The prostate-specific membrane antigen (PSMA) is highly expressed on most prostate cancer (PC) cells. Therefore, the targeting of PSMA has become increasingly important over the last decade. Glu-ureabased PSMA ligands used for both imaging and radioligand therapy are the mainstays of the current success. For PET imaging, both ${ }^{68} \mathrm{Ga}-$ and ${ }^{18} \mathrm{~F}$-labeled agents have been successfully translated to clinical applications. Mainly retrospective cohort studies have shown a high value in the setting of biochemical recurrence, with high detection rates even in the presence of low prostate-specific antigen levels. Preliminary data indicated that radioguided surgery with PSMA ligands may help to further improve patient outcomes because it facilitates the removal of small tumor deposits that are otherwise difficult to detect. For primary PC, PSMA ligand PET imaging has been shown to be superior to cross-sectional imaging for the detection of metastatic lymph nodes. In addition, it promises to also provide intraprostatic tumor localization, especially when used in combination with multiparametric MRI. Increasing numbers of studies have reported considerable changes in management resulting from PSMA ligand PET imaging for both biochemical recurrence and primary disease. The use of ${ }^{177}$ Lu-PSMA-based radioligand therapy has demonstrated a reasonable response, mainly as defined by a prostate-specific antigen response of more than $50 \%$, comparable to other recently introduced agents. Especially given the high level of safety of ${ }^{177}$ Lu-PSMA radioligand therapy, with only minimal grade 3 and 4 toxicities reported so far, it has the potential to expand options for metastatic castrationresistant PC. This review is intended to provide a comprehensive overview of the current literature on low-molecular-weight PSMA ligands for both PET imaging and therapeutic approaches, with a focus on agents that have been clinically adopted.

Key Words: prostate cancer; prostate-specific membrane antigen; imaging; therapy

J Nucl Med 2017; 58:67S-76S

DOI: 10.2967/jnumed.116.186767

Received Feb. 2, 2017; revision accepted Mar. 20, 2017.

For correspondence or reprints contact: Matthias Eiber, Department of Molecular and Medical Pharmacology, David Geffen School of Medicine, UCLA, 200 Medical Plaza, Suite B144, Los Angeles, CA 90095.

E-mail: matthias.eiber@tum.de

COPYRIGHT (C) 2017 by the Society of Nuclear Medicine and Molecular Imaging.
$\mathbf{P}$ rostate cancer (PC) is the most common malignancy worldwide in men, with persistently high numbers dying from this disease $(1,2)$. Imaging has increasingly been used over the last 2 decades to improve initial staging and help localize tumor deposits in recurrent disease. For instance, multiparametric MRI (mpMRI) has been shown to be effective in detecting or ruling out clinically significant disease, with a high negative predictive value of greater than $90 \%$ in various studies, and it can guide targeted biopsy $(3,4)$. Primary staging by bone scanning and cross-sectional imaging, as recommended for high-risk disease, is hampered by a high percentage of lymph node (LN) metastases in nonenlarged LNs (pooled sensitivity, $39 \%-42 \%)(5,6)$.

Approximately $30 \%-40 \%$ of patients will fail primary treatment, and a rising prostate-specific antigen (PSA) level will herald the onset of biochemical recurrence (BCR) (7). After potential salvage procedures (radiation and surgery), patients usually undergo androgen deprivation therapy. This treatment is typically followed by an increase in the PSA level after $2-8 \mathrm{y}$; this increase indicates the onset of metastatic castration-resistant PC (mCRPC) - the lethal form of the disease (8). Reactivation of androgen receptor signaling occurs in early mCRPC; therefore, second-line agents targeting the androgen receptor signaling axis have extended survival in clinical trials $(9,10)$. Treatment with other systemic agents, including taxane-based chemotherapy (e.g., docetaxel), sipuleucel-T, and the bone-seeking $\alpha$-emitter ${ }^{223} \mathrm{Ra}$, has been shown to improve overall survival and quality of life $(9,11-16)$. Nevertheless, patients with mCRPC have a poor prognosis and a median survival of 19 mo (17).

Molecular imaging has not played a major role until recently, as most PCs have only a low glycolytic rate, which limits the potential for ${ }^{18}$ F-FDG PET imaging $(18,19)$. Further advances have been achieved with choline derivatives. At BCR, a pooled detection rate of $62 \%$ was reported for ${ }^{11} \mathrm{C}$-choline PET/CT, but the rates were significantly lower when the PSA levels were low $(<1-2 \mathrm{ng} / \mathrm{mL})$ (20). At primary staging, the pooled sensitivity of choline PET/CT for the detection of LN metastases was low (49.2\%) in a recent metaanalysis (21). The results were similar when different cholinebased agents were compared (22). The intraprostatic localization of primary disease is hampered by nonspecific uptake in benign 
changes (e.g., benign prostatic hyperplasia) (23). Notably, choline derivatives and ${ }^{18} \mathrm{~F}$-FDG have shown potential in assessing the therapy response in mCRPC $(24,25)$. Most recently, 1 -amino$3-{ }^{18}$ F-fluorocyclobutane-1-carboxylic acid, an amino acid transport imaging agent, was approved by the U.S. Food and Drug Administration; however, its detection rate in recurrent PC is similar to those of choline derivatives $(26,27)$.

\section{UNMET CLINICAL NEEDS IN PC}

Accurate staging in primary PC and localization of early BCR for tailoring initial and subsequent treatment strategies are unmet clinical needs. Once mCRPC is diagnosed, effective therapy for improving overall survival and quality of life is desperately needed. Prostate-specific membrane antigen (PSMA) ligands are currently being investigated intensively, as they hold promise for extending the frontier in PC imaging and radioligand therapy (RLT). This review is intended to deliver a comprehensive overview of current and past investigations of low-molecular-weight PSMA radioligands for PC imaging and therapy. Providing insights into past and promising current developments regarding PSMA antibody imaging, especially targeting J591, is beyond the scope of this review.

\section{PSMA AS TARGET FOR PC IMAGING AND THERAPY}

PSMA is a type II transmembrane glycoprotein. PSMA expression and localization in the normal human prostate are associated with the cytoplasm and apical side of the epithelium surrounding the prostatic ducts (28). PSMA is enzymatically active only in its dimeric form, but its function for prostate cells is still unknown (29). Dysplastic and neoplastic transformation of prostate tissue results in the transfer of PSMA from the apical membrane to the luminal surface of the ducts $(30,31)$. For several reasons, PSMA is an ideal target in PC.

First, it is significantly overexpressed (100-1,000 fold) on nearly all PC cells (32-35), although 5\%-10\% of primary PC or PC lesions had negative PSMA results on PET $(36,37)$. However, the underlying etiology of PC with negative PSMA results on PET is still unclear, given the lack of prospective studies and correlation with immunohistochemistry.

Second, its expression is further increased in advanced stages and in mCRPC $(32,34,38)$.

Third, after binding to the active center of the extracellular domain, PSMA ligands are internalized. Subsequent endosomal recycling increases the deposition, leading to enhanced tumor uptake, retention, and subsequent high image quality for diagnostic procedures and a high local dose for therapeutic applications $(29,39,40)$.

Preclinical data showed that PSMA expression is independent of the use of taxane-based chemotherapy. This property may allow direct PC imaging — an advantage over bone scintigraphy and CT imaging-without the "flare phenomenon" (41). However, gene transcription analysis demonstrated that androgen can suppress the promoter of the PSMA gene $(42,43)$. Therefore, the initiation of androgen deprivation therapy induces early but temporary upregulation of PSMA expression, downregulation under prolonged androgen deprivation therapy and, finally, overexpression of PSMA in androgen-resistant tumors (44-47). A preliminary work outlined this effect, which could be leveraged for improved diagnosis and therapy (48).

Interestingly, PSMA expression in the tumor neovasculature of some solid tumors (colon, breast, kidney, and subtypes of bladder cancer) poses potential pitfalls in imaging $(32,49-54)$ but also offers potential opportunities for theranostic applications in cancers other than PC.

\section{COMPREHENSIVE OVERVIEW OF DIFFERENT CLASSES OF PSMA LIGANDS IN CLINICAL USE}

To date, no PSMA small molecule has been approved by the U.S. Food and Drug Administration or European Medicines Agency. The only approved agent targets the intracellular domain (7E11) of PSMA: the radiolabeled anti-PSMA antibody capromab pendetide (ProstaScint; EUSA Pharma) (35). Binding of the relatively large antibody to the intracellular domain of PSMA leads to false-positive findings after successful radiotherapy and very low rates of detection of viable tumor lesions (55).

In contrast, small-molecule PSMA ligands are relatively small peptide mimetic agents that are usually cleared rapidly from the circulation, leading to low background activities early after injection. The recognition of the structural and functional homology between $N$-acetyl-L-aspartyl-L-glutamate peptidase I and PSMA supported the development of these small molecules (56). The first class of PSMA-targeting radiopharmaceuticals based on phosphorous esters failed in early clinical translation (57). Other promising lead structures, such as carbamates, are still in preclinical evaluation (58). The focus of this review is the clinically most advanced category, the urea-based PSMA ligands, which usually consist of 3 components: the binding motif (glutamate-urea-lysine [Glu-urea-Lys] is the most widely used scaffold), a linker, and a radiolabel-bearing moiety (chelator molecule for radiolabeling or a prosthetic group for fluorinated agents) (Fig. 1).

Several of these agents have already been translated to clinical applications. Recent reviews described in detail the history and different characteristics of a broad spectrum of PSMA ligands, which is beyond the scope of this review (59-61). Table 1 provides an overview of the most commonly used low-molecular-weight PSMA ligands.

${ }^{123}$ I-MIP-1072 and ${ }^{123}$ I-MIP-1095 (62), the first agents, were followed by ${ }^{99 \mathrm{~m} T c-M I P-1404}$ and ${ }^{99 \mathrm{~m} T c-M I P-1405}$ (41) for SPECT imaging in clinical trials. $N-[N-[(S)-1,3$-dicarboxypropyl $]$

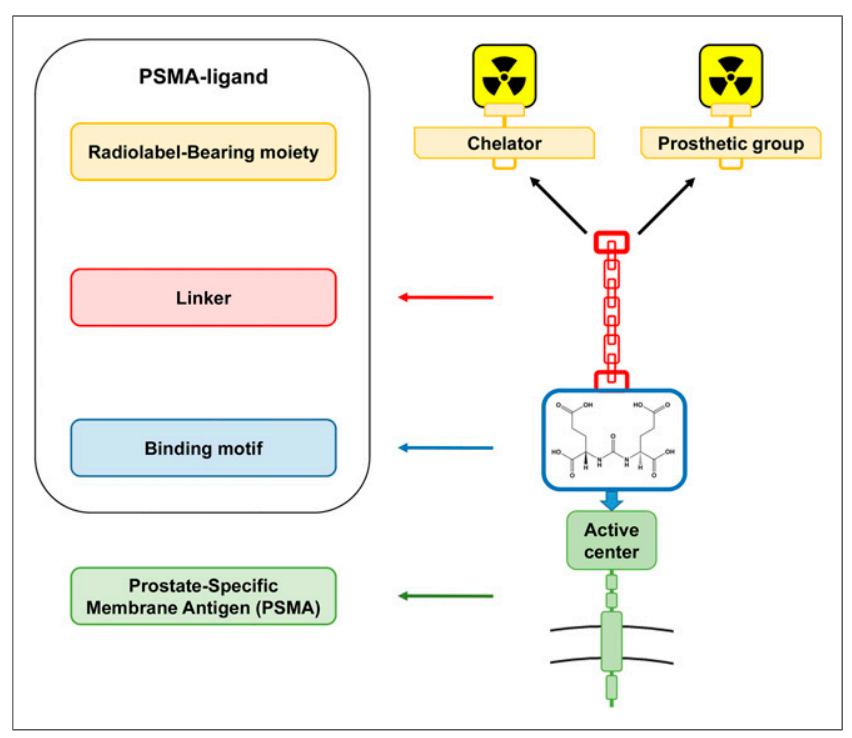

FIGURE 1. Structural components of urea-based PSMA ligands. 
TABLE 1

Overview of Most Commonly Used Glu-Urea-Based PSMA Ligands

\begin{tabular}{|c|c|c|}
\hline \multicolumn{2}{|c|}{ Chelator molecule } & \multirow[b]{2}{*}{ Prosthetic group molecule } \\
\hline Ligand & Label & \\
\hline PSMA-11 (PSMA-HBED-CC) & ${ }^{68} \mathrm{Ga}$ & ${ }^{18} \mathrm{~F}-\mathrm{DCFBC}$ \\
\hline \multicolumn{3}{|l|}{ Theranostic ligands } \\
\hline PSMA-617 & ${ }^{68} \mathrm{Ga},{ }^{177} \mathrm{Lu},{ }^{225} \mathrm{Ac}$ & ${ }^{18} \mathrm{~F}-\mathrm{DCFPyL}$ \\
\hline PSMA-I\&T & ${ }^{68} \mathrm{Ga},{ }^{177} \mathrm{Lu},{ }^{111} \mathrm{In}$ & ${ }^{18} \mathrm{~F}-\mathrm{PSMA}-1007$ \\
\hline MIP-1404 & 99mTc & 123|-MIP-1072, ${ }^{131}$ I-MIP-1095, 123I-MIP-1095 \\
\hline PSMA-I\&S & 99mTc & \\
\hline \multicolumn{3}{|c|}{$\begin{array}{l}\text { HBED-CC =N,N'-bis-[2-hydroxy-5-(carboxyethyl)benzyl]ethylenediamine- } N, N^{\prime} \text {-diacetic acid; PSMA-I\&S = PSMA for imaging and } \\
\text { surgery. } \\
\text { For theranostic ligands, only labels having published data are shown. }\end{array}$} \\
\hline
\end{tabular}

carbamoyl]-4- ${ }^{18}$ F-fluorobenzyl-L-cysteine $\left({ }^{18} \mathrm{~F}-\mathrm{DCFBC}\right)(63)$ and ${ }^{68} \mathrm{Ga}$-PSMA-11 ( ${ }^{68} \mathrm{Ga}$-PSMA- $N, N^{\prime}$-bis-[2-hydroxy-5-(carboxyethyl) benzyl] ethylenediamine- $N, N^{\prime}$-diacetic acid) (64) were the first agents for PET imaging. Initial therapeutic studies conducted with ${ }^{131}$ I-MIP-1095 (65) were soon followed by the introduction of chelator-based PSMA-617 (66) and another PSMA inhibitor for imaging and therapy, PSMA-I\&T (67), which are theranostic agents capable of being labeled with PET, SPECT, or therapeutic radionuclides. ${ }^{18} \mathrm{~F}$-labeled agents exploit an average lower positron energy leading to a higher intrinsic spatial resolution. Although ${ }^{18} \mathrm{~F}-$ DCFBC had persistently high blood-pool activity and relatively low tumor-to-background ratios, a second-generation, ${ }^{18} \mathrm{~F}$-labeled, small-molecule urea derivative known as 2-(3-\{1-carboxy-5-[(6${ }^{18}$ F-fluoro-pyridine-3-carbonyl)-amino]-pentyl $\}$-ureido)-pentanedioic acid $\left({ }^{18} \mathrm{~F}-\mathrm{DCFPyL}\right)$ overcame these limitations (68). Most recently, ${ }^{18}$ F-PSMA-1007 (69) was introduced as another ${ }^{18} \mathrm{~F}$-labeled agent exploiting very low urine clearance and potentially facilitating improved evaluation of the prostatic bed. ${ }^{18} \mathrm{~F}$-labeled agents also offer a longer half-life and potentially easier centralized production and distribution than ${ }^{68} \mathrm{Ga}$-labeled compounds (70). With a ${ }^{99 m}$ Tc-labeled PSMA for imaging and surgery, an alternative to the

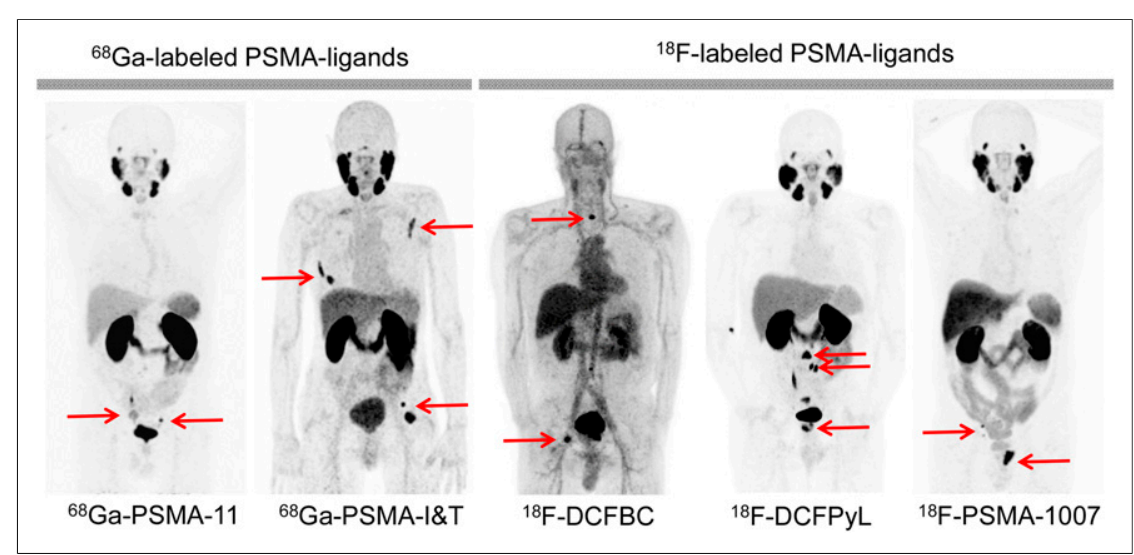

FIGURE 2. Maximum-intensity-projection images of PSMA ligands most commonly used for PET imaging ( ${ }^{68} \mathrm{Ga}$ - and ${ }^{18} \mathrm{~F}$-labeled agents). Images were acquired at different centers and show typical biodistribution as well as different numbers of positive lesions, depending on extent of disease in respective patients. Specific tumor uptake is indicated by red arrows. use of ${ }^{111}$ In for PSMA ligand-guided surgery is now available and may allow fast, convenient, kitlike labeling (71).

\section{PSMA LIGAND IMAGING}

\section{Biochemical Recurrence}

${ }^{68}$ Ga-Labeled PSMA Ligands. Since the initial report from 2012 (72), the most extensive experience with PSMA ligand PET imaging has been obtained for BCR. A recent metaanalysis based mainly on retrospective series summarized data for ${ }^{68} \mathrm{Ga}$-PSMA11 (73). Ten single, yet partially substantial, studies resulted in pooled detection rates of $58 \%$ and $76 \%$ for PSA levels of $0.2-1$ and 1-2 ng/mL, respectively (Figs. 2 and 3). The largest retrospective series of 319 patients ( 24 without prior definitive treatment) reported a detection rate of $88.1 \%$ on a per-patient basis (74). For lesions with histologic proof, a sensitivity of $76.6 \%$ and a specificity of $100 \%$ were demonstrated. In another study (75), a pooled detection rate of $89.5 \%$ was demonstrated for a homogeneous cohort of 248 patients after radical prostatectomy. Detection rates for early BCR at PSA levels of $0.2-0.5$ and $0.5-1 \mathrm{ng} / \mathrm{mL}$ were $57.9 \%$ and $72.7 \%$, respectively. In both studies, tumor detection was positively associated with PSA level but not with PSA doubling time.

In contrast, however, Ceci et al. (76) analyzed 39 patients with a PSA level of less than $2 \mathrm{ng} / \mathrm{mL}$ and reported a strong relationship with PSA doubling time: The detection rates were $85 \%$ for a PSA doubling time of less than 6.5 mo but only $19 \%$ for a PSA doubling time of greater than 6.5 mo. For ${ }^{68} \mathrm{Ga}$-PSMA-I\&T, an initial study (77) reported detection rates of $52 \%$ for a PSA level of less than $0.5 \mathrm{ng} / \mathrm{mL}, 55 \%$ for $0.5-1.0 \mathrm{ng} /$ $\mathrm{mL}, 70 \%$ for $1.0-2.0 \mathrm{ng} / \mathrm{mL}$, and $93 \%$ for $2.0-5.0 \mathrm{ng} / \mathrm{mL}$; these results were relatively comparable to those for ${ }^{68} \mathrm{Ga}-\mathrm{PSMA}-11$, although they were limited by the small number of patients $(n=83)$.

Several studies demonstrated the superiority of ${ }^{68} \mathrm{Ga}$-PSMA-11 over choline derivatives for PET/CT in a head-to-head comparison of 


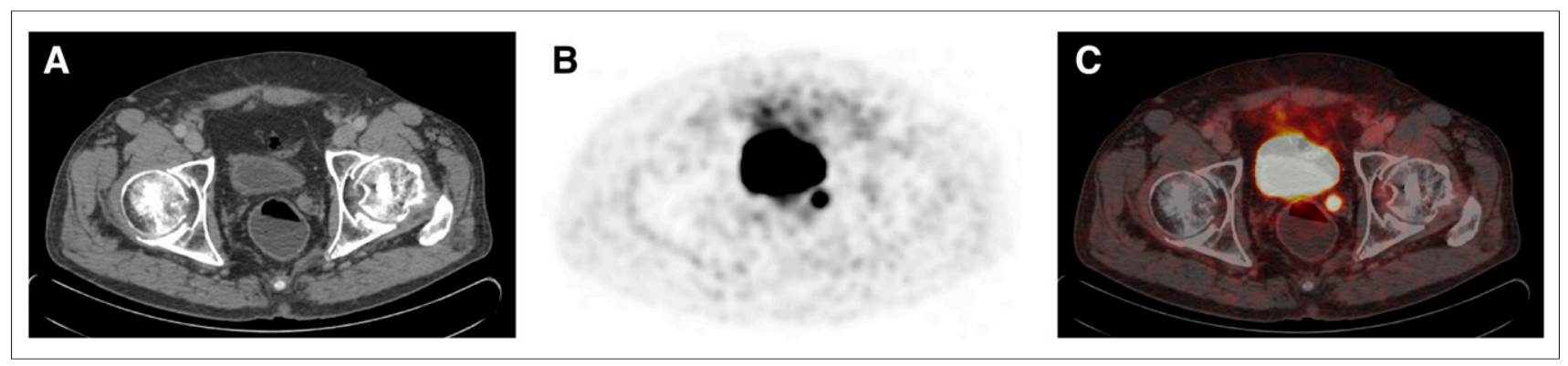

FIGURE 3. 76-y-old patient with BCR (PSA of $0.4 \mathrm{ng} / \mathrm{mL}$ ) after radical prostatectomy (initial stage, pT2c pNo G2; Gleason score of $7 \mathrm{~b}$ ). Patient was referred for ${ }^{68} \mathrm{Ga}-\mathrm{PSMA}-11 \mathrm{PET} / \mathrm{CT}$ for restaging. ${ }^{68} \mathrm{Ga}-\mathrm{PSMA}-11 \mathrm{PET} / \mathrm{CT}$ showed local recurrence in left seminal vesicle bed. (A) Transaxial CT. (B) Transaxial PET. (C) Transaxial fused PET/CT.

patients with BCR (78-80). Afshar-Oromieh et al. detected PC in 32 of 37 BCR patients $(86.5 \%)$ with ${ }^{68} \mathrm{Ga}-\mathrm{PSMA}-11$ but in only 26 of 37 patients $\left(70.3 \%\right.$ ) with ${ }^{18} \mathrm{~F}$-fluorocholine (80). Both the $\mathrm{SUV}_{\max }$ and tumor-to-background ratios were superior for ${ }^{68} \mathrm{Ga}$-PSMA-11 in most lesions (79\% and 95\%, respectively). In a study by Morigi et al., 14 of 26 patients $(53.8 \%)$ had positive results only in ${ }^{68} \mathrm{Ga}$-PSMA-11 PET/ CT, 1 patient (4\%) had positive results only in ${ }^{18} \mathrm{~F}$-fluoromethylcholine $\mathrm{PET} / \mathrm{CT}$, and the remaining patients were diagnosed by consensus in both investigations (78).

There is increasing evidence that a patient's prognosis is improved by the initiation of salvage therapy before the PSA level exceeds $0.5 \mathrm{ng} / \mathrm{mL}$ (17). For such patients, the rate of detection by ${ }^{68} \mathrm{Ga}$-PSMA-11 PET/CT in various studies was reported to be between $50.0 \%$ and $57.9 \%(74,75,78,81)$-higher than for any other imaging modality. Thus, ${ }^{68} \mathrm{Ga}$-PSMA-11 PET/CT potentially allows for more effective salvage treatment, including radiotherapy with focal boost and salvage node dissection. Further evidence is needed to ascertain whether this treatment improves patient outcomes, but modifying management rather than administering blind radiotherapy to the prostate bed in patients with occult extraprostatic disease identified by PSMA PET/CT is a logical approach.

${ }^{18} \mathrm{~F}$-Labeled PSMA Ligands. A first-in-man study of ${ }^{18} \mathrm{~F}-\mathrm{DCFBC}$ in 5 patients with metastatic disease revealed favorable dosimetry and biodistribution for the detection of PC (63). In addition, putative sites of occult disease on conventional imaging were detected. A small prospective study of 17 patients with known progressive metastatic PC (both patients with hormone-naive disease and those with castration-resistant disease) demonstrated the superiority of ${ }^{18} \mathrm{~F}$ DCFBC PET/CT over conventional imaging with CT and bone scanning for lesion detection (82).

A first-in-man analysis of ${ }^{18} \mathrm{~F}-\mathrm{DCFPyL}$ in 9 patients with known metastatic PC determined favorable dosimetry, biodistribution, and safety (83). Subsequent analyses demonstrated markedly increased lesion detection efficiency relative to that of conventional imaging (84). In a head-to-head comparison of 14 selected patients who underwent ${ }^{18} \mathrm{~F}$-DCFPyL PET/CT after ${ }^{68} \mathrm{Ga}$-PSMA-11 PET/CT because of negative or inconclusive findings, ${ }^{18} \mathrm{~F}-\mathrm{DCFPyL} \mathrm{PET} / \mathrm{CT}$ revealed additional lesions in 3 of 14 patients (21.4\%) (85). In addition, the mean $\mathrm{SUV}_{\max }$ in concordant ${ }^{18} \mathrm{~F}-\mathrm{DCFPyL}$ PSMApositive lesions was significantly higher than that obtained with ${ }^{68}$ Ga-PSMA-11 (14.5 vs. $\left.12.2 ; P=0.028 ; n=15\right)$. In a follow-up study by this group, PSA-stratified detection rates in different patients ( $n=62$ with ${ }^{18} \mathrm{~F}$-DCFPyL and $n=129$ with ${ }^{68} \mathrm{Ga}$-PSMA-11) were compared (86). These initial data in a limited number of patients suggested that ${ }^{18} \mathrm{~F}$-DCFPyL was not inferior to ${ }^{68} \mathrm{Ga}$-PSMA-11 and indicated that imaging with ${ }^{18} \mathrm{~F}-\mathrm{DCFPyL}$ might even exhibit im- proved sensitivity in localizing relapsed tumors after prostatectomy for moderately increased PSA levels. However, further conclusions are difficult to draw because of the different patient populations, administered activities, and PET acquisition techniques.

The potential of ${ }^{18}$ F-PSMA-1007 to detected morphologically occult disease was demonstrated in a recent case report (87). Lesions had an $\mathrm{SUV}_{\text {median }}$ and an $\mathrm{SUV}_{\text {max }}$ of 7.7 and 18.8, respectively.

PSMA Ligands for SPECT Imaging. Only a few studies have investigated the use of SPECT agents in recurrent PC. An initial study of ${ }^{99 m}$ Tc-MIP-1404 in 6 healthy men and 6 patients with radiographic evidence of metastatic PC showed favorable biodistribution, identified most bone metastases (compared with bone scintigraphy), and detected soft-tissue PC lesions, including subcentimeter LNs (88). Subsequently, in an evaluation of $99 \mathrm{~m}$ Tc-MIP1404 in 60 patients, the detection rates at PSA levels of greater than $2 \mathrm{ng} / \mathrm{mL}$ and less than or equal to $2 \mathrm{ng} / \mathrm{mL}$ were $91.4 \%$ and $40.0 \%$, respectively (89). A direct comparison of ${ }^{111} \mathrm{In}$-PSMA-I\&T and ${ }^{68} \mathrm{Ga}-$ PSMA-11 in patients with early recurrence clearly showed a lower detection rate for ${ }^{111}$ In-PSMA-I\&T (90). Only 14 of 29 lesions with PET-positive results $(48.3 \%)$ were visualized by ${ }^{111}$ In-PSMA I\&T.

Impact on Clinical Decisions. Evidence that PSMA ligand PET imaging significantly affects management in patients with early BCR is increasing. In a homogeneous cohort of 70 patients who had a PSA level of less than $1 \mathrm{ng} / \mathrm{mL}$ and were potential candidates for salvage radiation treatment, ${ }^{68} \mathrm{Ga}$-PSMA-11 PET/CT led to a major change in management (disease in LN or bone not covered by the conventional radiation therapy field) for $28.6 \%$ of patients (81). In an additional $26 \%$ of patients, the disease was confined to the prostate bed, possibly defining these patients as optimal candidates for salvage radiation therapy. Morigi et al. prospectively evaluated the impact on management of ${ }^{68} \mathrm{Ga}$-PSMA-11 in patients with BCR and prior ${ }^{18} \mathrm{~F}$-fluorocholine treatment; they demonstrated a major impact in approximately one-third of patients, especially those with a low PSA level (78). Two other studies reported similar results $(91,92)$. Data are limited in patients with metastatic disease. The potential of PSMA PET/CT to stratify and monitor advanced disease is unclear (Fig. 4). A preliminary report indicated that ${ }^{68} \mathrm{Ga}$-PSMA-11 might be a useful gatekeeper before ${ }^{223}$ Ra-dichloride therapy (93).

\section{Primary Staging}

${ }^{68}$ Ga-Labeled PSMA Ligands. Several studies have focused on the accuracy of ${ }^{68} \mathrm{Ga}-\mathrm{PSMA}-11 \mathrm{PET} / \mathrm{CT}$ for primary LN staging (Fig. 5). In a retrospective analysis of 130 consecutive patients with primary intermediate- to high-risk PC and subsequent template-based pelvic LN dissection, ${ }^{68}$ Ga-PSMA-11 PET outperformed cross-sectional imaging at the patient and template 


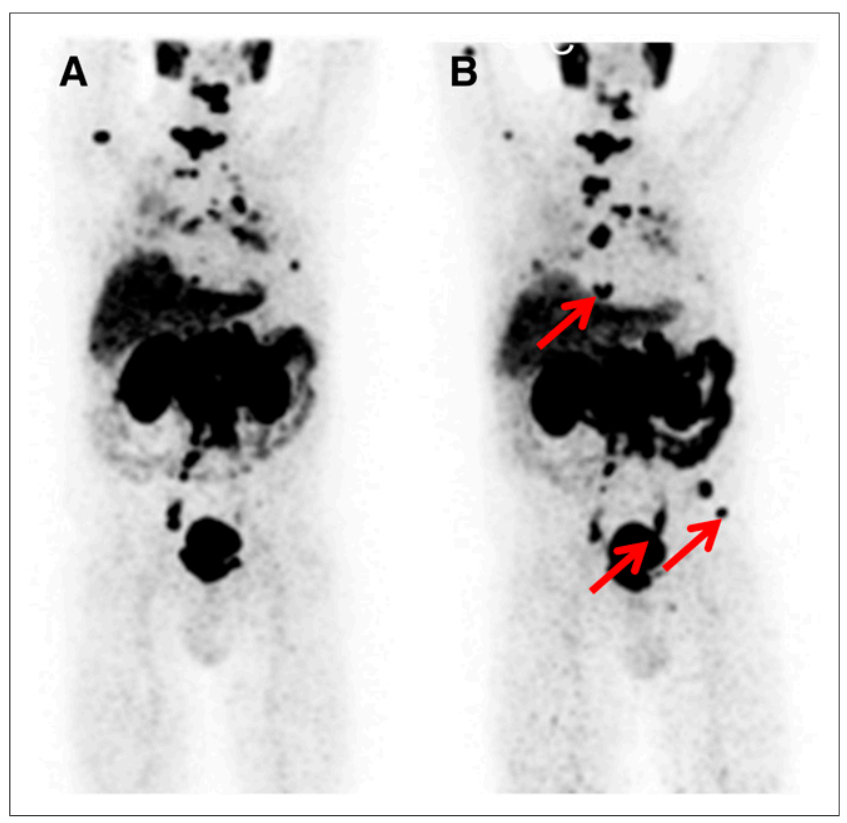

FIGURE 4. 73-y-old patient with mCRPC (Gleason score of 9). Patient received systemic treatment and was referred for ${ }^{68} \mathrm{Ga}$ PSMA-11 PET/ $\mathrm{CT}$ before and after docetaxel chemotherapy for therapy response assessment. (A) Baseline PET/CT revealed PSMA-positive disseminated bone, nodal, and lung metastases (maximum-intensity projection [MIP]). (B) Follow-up PET/CT showed mixed response, with evidence of new metastases (red arrows) (MIP). Accordingly, PSA level increased from $343 \mathrm{ng} / \mathrm{mL}$ at baseline to $455 \mathrm{ng} / \mathrm{mL}$ at follow-up.

levels ( $P=0.002$ and $P<0.001$, respectively). Reported values for the sensitivity of ${ }^{68} \mathrm{Ga}$-PSMA-11 PET/CT versus conventional imaging were $65.9 \%$ versus $43.9 \%$, respectively, at the patient level and
$68.3 \%$ versus $27.3 \%$, respectively, at the template level. Moreover, ${ }^{68}$ Ga-PSMA-11 PET had a high specificity-greater than $95 \%$. Nine patients with LNs found positive by histopathology but negative by ${ }^{68} \mathrm{Ga}$-PSMA-11 PET harbored only micrometastases in single LNs (mean size of negative LNs, $3 \mathrm{~mm}$ ). Impaired detection of LN metastases smaller than $5 \mathrm{~mm}$ was anticipated and was also reported by other groups with similar results for the overall performance of ${ }^{68} \mathrm{Ga}-\mathrm{PSMA}-11$ PET for node staging $(81,94,95)$.

For the identification of bone metastases, ${ }^{68} \mathrm{Ga}-\mathrm{PSMA}-11$ PET was clearly superior to bone scanning in a preliminary comparison that also explored primary PC on both per-patient and per-region bases ( $P=0.006$ and $P<0.0001$, respectively) (96). A prospective phase 3 multicenter trial comparing ${ }^{68} \mathrm{Ga}$-PSMA-11 PET with conventional imaging (CT and bone scanning) in men with newly diagnosed Gleason grade group 3 to $5 \mathrm{PC}$ is currently under way (ANZCTR 12617000005358).

Increasing numbers of studies have investigated the potential of ${ }^{68}$ Ga-PSMA-11 for intraprostatic tumor localization (97-102). For example, on the basis of a 6-segment model per slice, histopathologically positive segments ( $n=100$ in 21 patients) had significantly higher ${ }^{68} \mathrm{Ga}-\mathrm{PSMA}-11$ uptake than negative segments $\left(\mathrm{SUV}_{\max }, 11.8\right.$ vs. 4.9), suggesting an $\mathrm{SUV}_{\max }$ of 6.5 as an optimal discriminator (sensitivity of $67 \%$ and specificity of $92 \%$ for the detection of tumor-involved segments) (97). Other retrospective studies investigating 6 and 9 patients and using segment and voxelwise approaches reported similar results $(98,101)$.

${ }^{68}$ Ga-PSMA-11 PET was compared head-to-head with mpMRI, the current standard method for intraprostatic tumor localization, in several studies. Giesel et al. analyzed the concordance between the methods (102). The nearly total agreements between positive findings on one modality and a positive correlate on the other were $89.4 \%$ for mpMRI and $96.8 \%$ for ${ }^{68} \mathrm{Ga}$-PSMA-11 PET; these results indicated a potential benefit of a combination of the methods for

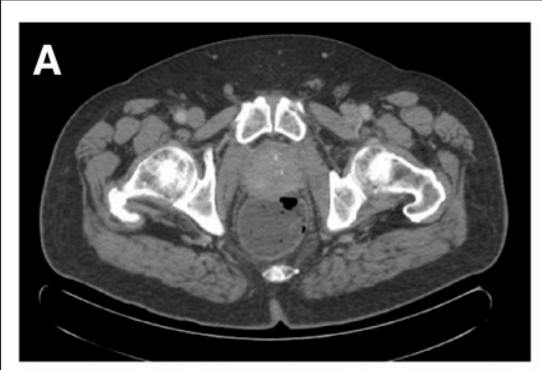

B

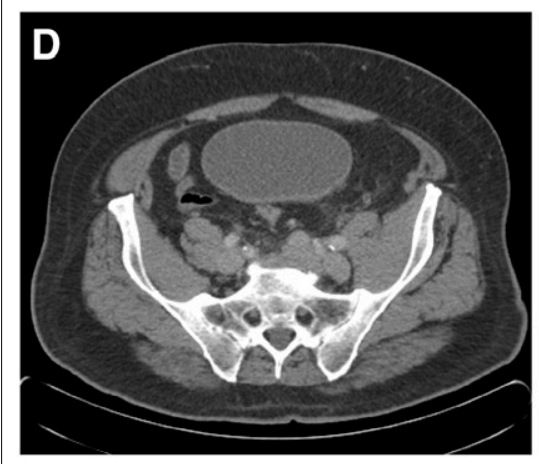

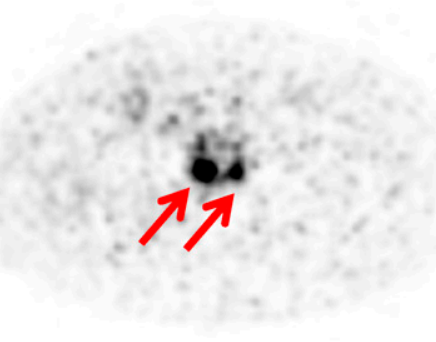

E

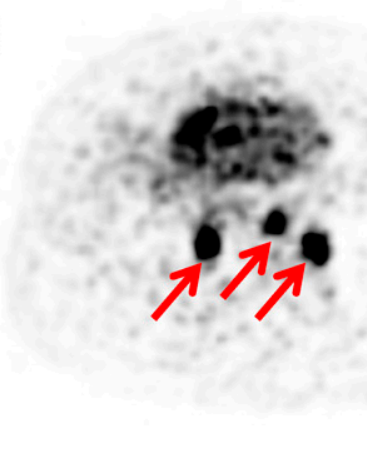

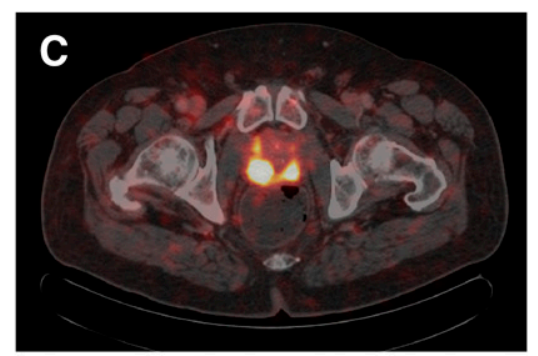

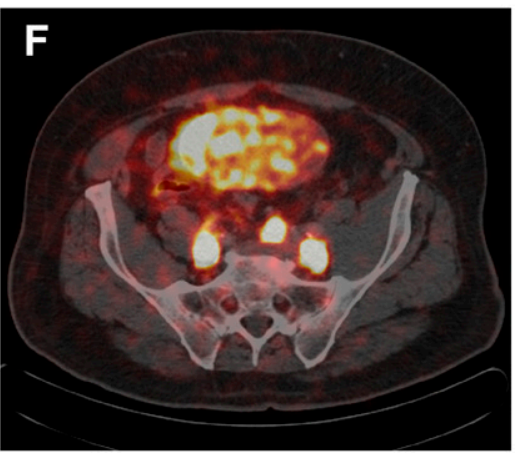

FIGURE 5. 70-y-old patient with biopsy-proven high-risk PC (Gleason score of 9; PSA of $94 \mathrm{ng} / \mathrm{mL})$. Patient was referred for ${ }^{68} \mathrm{Ga}-\mathrm{PSMA}-11 \mathrm{PET} /$ CT for primary staging. ${ }^{68} \mathrm{Ga}-\mathrm{PSMA}-11 \mathrm{PET} / \mathrm{CT}$ showed PSMA-positive primary tumor (red arrows) (transaxial CT [A], PET [B], and fused PET/CT [C]) and pelvic LN involvement on both sides (red arrows) (transaxial CT [D], PET [E], and fused PET/CT [F]). 
tumor detection. The use of combined ${ }^{68} \mathrm{Ga}$-PSMA-11 PET/MRI was analyzed in 53 patients with intermediate- and high-risk PC (99). In a sextant-based analysis of the whole prostate gland, a higher accuracy was found for combined mpMRI and ${ }^{68} \mathrm{Ga}$-PSMA-11 PET. Sensitivities and specificities were $76 \%$ and $97 \%$, respectively, for hybrid ${ }^{68} \mathrm{Ga}$-PSMA-11 PET/MRI; $58 \%$ and $82 \%$, respectively, for mpMRI $(P=0.003)$; and $64 \%$ and $94 \%$, respectively, for ${ }^{68} \mathrm{Ga}-$ PSMA-11 PET. Potential implications include improvement of biopsy guidance or radiation treatment planning.

Zamboglou et al. investigated the gross tumor volume based on mpMRI, ${ }^{68} \mathrm{Ga}$-PSMA-11 PET, and the combination in 7 patients (101). The gross tumor volume based on PET plus mpMRI had a significantly higher overlap $(57 \% \pm 22 \%$ [mean $\pm \mathrm{SD}])$ than did the gross tumor volume based on mpMRI alone $(42 \% \pm 18 \%)$ or ${ }^{68}$ Ga-PSMA PET alone $(45 \% \pm 24 \%)$ when the data were validated by histopathology. Interestingly, each lesion with the highest uptake in PET corresponded to the lesion with the highest diffusion restriction (lowest apparent diffusion coefficient) in mpMRI.

${ }^{18}$ F-Labeled PSMA Ligands. In a study of 13 patients who underwent ${ }^{18} \mathrm{~F}$-DCFBC PET/CT before prostatectomy, 12 had contemporaneous pelvic MRI for comparison (103). Although the sensitivity of MRI was superior to that of ${ }^{18} \mathrm{~F}$-DCFBC PET/CT imaging in this cohort, ${ }^{18} \mathrm{~F}-\mathrm{DCFBC}$ PET/CT had a high specificity for the most clinically significant high-Gleason score and high-volume tumors. Furthermore, a correlation between the degree of radiotracer uptake and the Gleason score of the dominant tumor was noted, although the small number of patients contributed to wide confidence intervals (103) — in contrast to a larger patient cohort imaged with ${ }^{68}$ Ga-PSMA-11 (99). Unlike the results obtained with many nonPSMA-targeted radiotracers, foci of benign prostatic hyperplasia were photopenic when imaged with ${ }^{18} \mathrm{~F}$-DCFBC PET/CT (103). Preliminary data for ${ }^{18} \mathrm{~F}-\mathrm{PSMA}-1007$ indicated a high accuracy for the detection of nodal involvement in primary disease (69). Most affected LNs (18/19 LN metastases in a total of 309 resected LNs), including nodes as small as $1 \mathrm{~mm}$ in diameter, were identified correctly.

PSMA Ligands for SPECT Imaging. In a phase 2 clinical trial, ${ }^{99 \mathrm{~m} T c-M I P-1404}$ had a sensitivity of $94 \%$ and a specificity of $100 \%$ for the detection of primary $\mathrm{PC}$ in a preselected high-risk population. Its clinical impact is currently being evaluated in a prospective phase 3 clinical trial (ClinicalTrials.gov identifier: NCT02615067).

Impact on Clinical Decisions. The decision to use primary radiotherapy or radical prostatectomy either with or without extended pelvic lymphadenectomy or radiation of lymphatic drainage is challenging and depends mainly on patient as well as pretherapeutic tumor characteristics. Current approaches use epidemiological data to pretherapeutically estimate the baseline risk for diseasepositive nodes $(104,105)$. In a recent publication, ${ }^{68} \mathrm{Ga}$-PSMA-11 $\mathrm{PET} / \mathrm{CT}$ and standard-of-care imaging (including bone scanning, $\mathrm{CT}$, and MRI) were performed in 57 patients for primary radiotherapy. Imaging led to modifications in $50.8 \%$ of patients (91). The specific impact of ${ }^{68} \mathrm{Ga}-\mathrm{PSMA}-11$ PET on planning for local radiation was also indicated in a study involving mpMRI comparison. Besides a $47 \%$ concordance of PET and mpMRI for identifying the dominant intraprostatic lesion at the patient level, the PET-based gross tumor volume was significantly larger (106).

\section{PSMA LIGAND-BASED THERAPEUTIC APPLICATIONS}

\section{Radionuclide Therapy}

The radiolabeled anti-PSMA antibody ${ }^{177} \mathrm{Lu}$-J591, which targets the extracellular domain of PSMA, resulted in any PSA decline in
$60 \%$ of 47 patients included in the study; however, only $11 \%$ experienced a decline in the PSA level of greater than $50 \%$, and treatment was limited by hematologic toxicity, with grade 4 thrombocytopenia occurring in $47 \%$ (107). The slow diffusion of antibodies into solid lesions, with an uptake maximum at 6 or $7 \mathrm{~d}$ after injection, and hematotoxicity caused by a long circulation time in blood are significant limitations of radiolabeled antibody therapy (108). Small-molecule PSMA ligands for endoradiotherapy were first used by Zechmann et al., who treated 28 mCRPC patients with a single therapeutic dose of ${ }^{131}$ I-MIP-1095 (mean activity, $4.8 \mathrm{GBq}$; range, $2-7.2 \mathrm{GBq}$ ) (65). The retention of ${ }^{131} \mathrm{I}$-MIP-1095 in lesions was visualized up to $17 \mathrm{~d}$ after a single dose, and considerable symptom relief and a decline in the serum PSA level of greater than $50 \%$ were noted in more than half of the patients.

\section{Safety and Efficacy of ${ }^{\mathbf{1 7 7}}$ Lu-Labeled PSMA Ligands}

${ }^{177} \mathrm{Lu}$ offers several advantages over ${ }^{131} \mathrm{I}$, such as easier distribution to therapy centers (less heavy shielding needed because of the lower $\beta$-particle energy: 133 vs. $182 \mathrm{keV}$ ), easier radiolabeling, and a lower proportion of $\gamma$-radiation-potentially resulting in reduced hospital admission times and lower toxicity (109). The German Society of Nuclear Medicine recently published a consensus recommendation on the use of ${ }^{177} \mathrm{Lu}$-PSMA-617 in mCRPC patients (110). Most retrospective case series for PSMA-targeted RLT involved ${ }^{177}$ Lu-PSMA-617 (111-115). Most studies showed a decrease in the PSA level of greater than or equal to $50 \%$ in $30 \%-60 \%$ of mCRPC patients (Fig. 6). Side effects seemed to be relatively mild, with only a few cases of grade 3 and no grade 4 nonhematologic toxicity being reported. However, long-term results regarding potential toxicities to the salivary glands and kidneys, given the high levels of physiologic accumulation, are still pending.

The largest multicenter retrospective case series for toxicity and PSA response in patients after ${ }^{177} \mathrm{Lu}$-PSMA-617 treatment was published as part of an initiative by the German Society of Nuclear Medicine (110). Data were collected for 145 patients who had mCRPC and had undergone a median of 2 cycles (range, 1-4) of ${ }^{177} \mathrm{Lu}$-PSMA-617 RLT at 12 centers. Most patients received 5.5-6.5 $\mathrm{GBq}$ (median, 6.0 GBq) or greater than $6.5 \mathrm{GBq}$ (median, 7.4 GBq) per cycle. The rate of nonhematologic grade 3 or 4 events was low ( 0 3 patients; $0 \%-2 \%$ ). Grade 3 or 4 anemia occurred in 15 patients (10\%), and grade 3 or 4 thrombocytopenia occurred in 5 patients $(4 \%)$.

A decline in the PSA level of greater than or equal to $50 \%$ was seen in 45 of 99 patients ( $45 \%$ ) over the entire follow-up period. Any decline in the PSA level occurred in 59 of 99 patients (60\%). After the first therapy cycle, a decline in the PSA level of greater than or equal to $50 \%$ was observed in 40 of 99 patients (40\%), and any decline in the PSA level was observed in 65 of 99 patients $(66 \%)$. After the second therapy cycle, a decline in the PSA level of greater than or equal to $50 \%$ was seen in 35 of 61 patients $(57 \%)$, and any decline in the PSA level was seen in 44 of 61 patients (72\%). Most patients who received a third or fourth cycle showed a decline in the PSA level of greater than or equal to $50 \%(65 \%$ or $100 \%$, respectively). A potential limitation of that study (110) was the strict date for data inclusion, which omitted an analysis of follow-up data as well as inclusion of further cycles in patients still under treatment.

Baum et al. published a study of the largest series of patients with ${ }^{177}$ Lu-PSMA-I\&T RLT (112). A total of 125 cycles (up to 5 cycles per patient; median administered activity, 5.8 GBq) were administered in 56 patients. A decrease in the serum PSA level was reported in $80.3 \%$ of patients. The median progression-free survival was $13.7 \mathrm{mo}$, and the median overall survival was not 

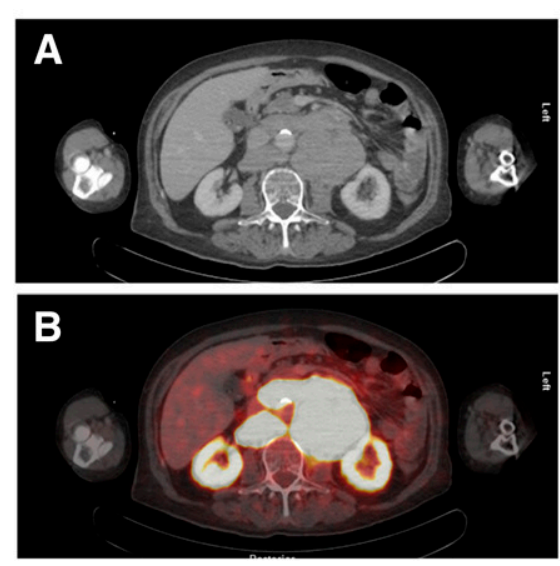
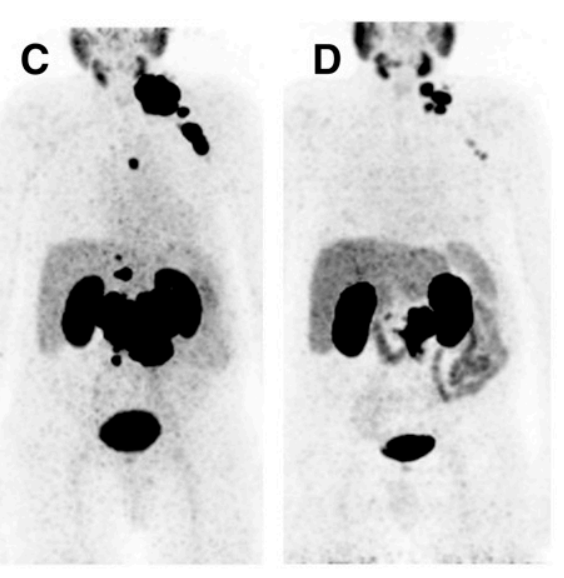
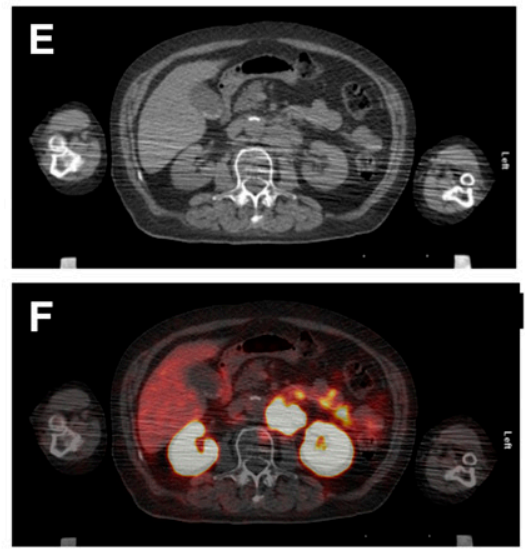

FIGURE 6. 77-y-old patient with mCRPC (initial stage, cT3a cN1 cM0 G2b; Gleason score of 7a) before (A-C) and after (D-F) ${ }^{177}$ Lu-PSMA-617 RLT. Patient received androgen deprivation therapy, local irradiation, and several lines of systemic therapy. Because of progression, patient was referred for 3 cycles of ${ }^{177}$ Lu PSMA-617 therapy. ${ }^{68} \mathrm{Ga}-\mathrm{PSMA}-11$ PET/CT demonstrated extensive PSMA-positive nodal metastases at baseline (transaxial CT [A], fused PET/CT [B], and maximum-intensity projection [MIP] [C]) and reduced tumor load after 3 cycles of ${ }^{177}$ Lu-PSMA-617 therapy (MIP [D], transaxial CT [E], and fused PET/CT [F]). PSA level dropped from $291 \mathrm{ng} / \mathrm{mL}$ before ${ }^{177} \mathrm{Lu}-\mathrm{PSMA}-617$ therapy to $38 \mathrm{ng} / \mathrm{mL}$ after ${ }^{177} \mathrm{Lu}-$ PSMA-617 therapy.

reached at the follow-up of 28 mo. Heck et al. reported the safety and efficacy of ${ }^{177}$ Lu-PSMA-I\&T in mCRPC patients (116). No grade 3 or 4 toxicity was reported. Grade 1 or 2 xerostomia occurred in 7 patients (37\%), anemia occurred in 6 patients $(32 \%)$, and thrombocytopenia occurred in 5 patients $(25 \%)$. A maximum decline in the PSA level of greater than or equal to $50 \%$ was achieved in $33 \%$ of patients. The Eastern Cooperative Oncology Group performance status improved or was stable in $74 \%$ of patients. Complete resolution or reduced pain after treatment was achieved in $58 \%$ of patients with bone pain.

\section{Dosimetry}

Several dosimetric analyses for both ${ }^{177}$ Lu-PSMA-617 and ${ }^{177}$ Lu-PSMA-I\&T have been published (112,117-120). They documented favorable dosimetry with a low red marrow doseapproximately $0.03 \mathrm{~Gy} / \mathrm{GBq}$. Organs at risk are the kidneys $(0.5-$ $0.7 \mathrm{~Gy} / \mathrm{GBq})$ and the salivary or lacrimal glands (1.2-2.8 Gy/ $\mathrm{GBq}$ ). However, universal limits for a cumulative dose by endoradiotherapy have not been established, and radiation-induced nephropathy or severe xerostomia has been observed only in individual cases thus far. Tumor doses were estimated to be between

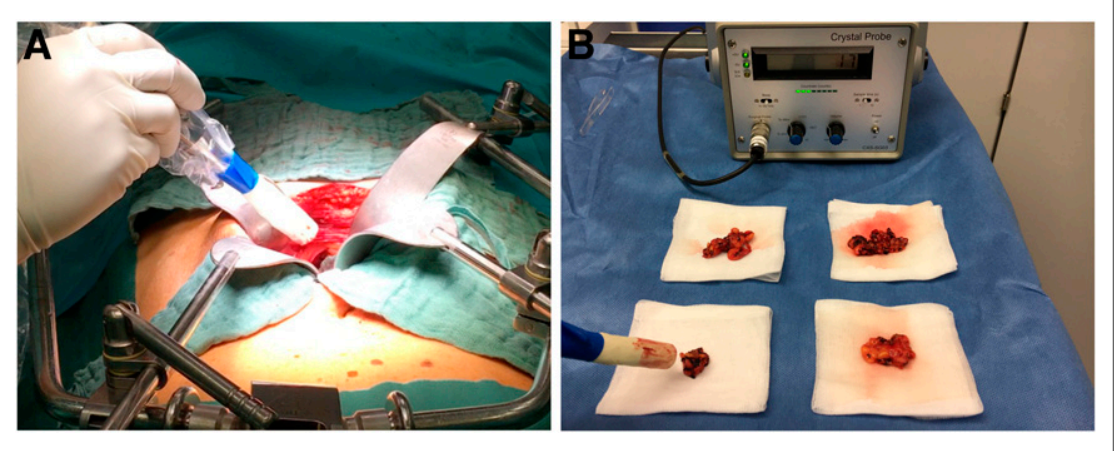

FIGURE 7. PSMA RGS. In vivo (A) and ex vivo (B) intraoperative y-probe measurements facilitated complete resection of PSMA ligand-positive LN prior to detection on ${ }^{68} \mathrm{Ga}-\mathrm{PSMA}-11$ PET/CT.
1.2 and 47.5 Gy/GBq (mean, 13.1 Gy/GBq). Despite high tumor doses, all authors reported that some patients (20\%-40\%) were refractory to treatment with ${ }^{177} \mathrm{Lu}$-PSMA ligands even though there was sufficient PSMA expression on pretherapeutic PET. Patients with diffuse red marrow infiltration, which was considered a risk factor for the development of a higher level of hematotoxicity (118), presented another challenge. A promising option for overcoming this limitation could be the application of PSMA ligands labeled with the short-range $\alpha$-particle emitter ${ }^{225}$ Ac. Kratochwil et al. reported a promising response with ${ }^{225}$ Ac-PSMA-617 in 2 patients who showed progression after ${ }^{177}$ Lu-PSMA-617 treatment (121). However, in that initial experience, substantial xerostomia was a side effect.

\section{Radioguided Surgery (RGS)}

Improved tumor localization with PSMA ligands now allows the detection in early BCR of tiny and often atypically located lesions that often cannot be visualized reliably during surgery (Fig. 7). Maurer et al. attempted to expand the spectrum of radiolabeled PSMA ligands toward RGS using ${ }^{111}$ In-labeled PSMAI\&T (122). One patient with primary disease and 4 patients with $\mathrm{BCR}$ and evidence of solely regional $\mathrm{LN}$ metastases only on ${ }^{68}$ Ga-PSMA-11 PET underwent RGS. Patients received a mean of $146 \mathrm{MBq}$ (range, 110-169 MBq) of ${ }^{111}$ In-PSMA-I\&T $24 \mathrm{~h}$ before surgery. In that feasibility study, all tissue specimens that showed positive radiosignals in vivo were confirmed to bear PSMA-expressing metastatic disease by ex vivo histopathology (Fig. 7). The detection of subcentimeter lesions was enabled by RGS. In 2 patients, tumor deposits that were not visualized on preoperative ${ }^{68} \mathrm{Ga}-\mathrm{PSMA}-11$ PET imaging were detected by RGS.

A follow-up study summarized the data for 31 patients after salvage PSMA RGS (123). Declines in the PSA level of greater 
than $50 \%$ and greater than $90 \%$ were observed in 23 of 30 patients (76.7\%) and 16 of 30 patients $(53.3 \%)$, respectively. In 18 of 30 patients $(60.0 \%)$, a decline in the PSA level to less than $0.2 \mathrm{ng} / \mathrm{mL}$ was achieved. In 10 of 30 patients (33.3\%), further PC-specific treatment was given after a median of $125 \mathrm{~d}$ (range, 48-454 d) following ${ }^{111}$ In-PSMA-I\&T RGS. The remaining 20 patients were treatment-free at a median follow-up of $337 \mathrm{~d}$ (range, 81-591 d). These data indicate that ${ }^{111}$ In-PSMA I\&T RGS might have a beneficial influence on further disease progression; however, the identification of suitable patients on the basis of PSMA PET as well as clinical variables is essential for satisfactory results. A potential limitation of RGS is the missing direct visualization of tumor tissue in real time. At present, researchers are working on PSMA ligands suitable for real-time near-infrared fluorescence imaging of surgical margins and fluorescence-guided resection of tumor residues (124).

\section{CONCLUSION}

PSMA ligands for both imaging and therapy have been adopted clinically at an unprecedented rate, resulting in dramatic increases in the number of research publications. Current evidence from the literature strongly indicates that PSMA ligand PET imaging may have considerable influence on the management of early recurrent disease as well as primary (intermediate- and high-risk) PC. In addition, therapeutic approaches, mainly with ${ }^{177} \mathrm{Lu}$-PSMA RLT, have been shown to be valuable for the treatment of advanced disease; further advances are to be expected from the use of $\alpha$-emitters. However, current data rely predominantly on retrospective analyses. Well-defined patient cohorts and dedicated endpoints are needed for the implementation of these technologies into guidelines. Fortunately, several academic as well as industrydriven studies are already under way or planned and may provide the needed evidence. However, because of the multitude of PSMA ligands as well as the different approaches chosen, predicting which agents may finally reach regulatory approval and clinical reimbursement is difficult. For the well-being of their patients, both nuclear medicine physicians and their clinical counterparts must jointly create algorithms for the wise use of these new tools.

\section{DISCLOSURE}

Clemens Kratochwil and Frederik L. Giesel disclose a patent application (EP 15002 800.9, DKFZ) for PSMA-1007. No other potential conflict of interest relevant to this article was reported.

\section{ACKNOWLEDGMENTS}

We thank Thorsten Derlin for contributing a case of ${ }^{68} \mathrm{Ga}-$ PSMA-I\&T PET/CT imaging as well as Christine Mona for assisting in designing figures.

\section{REFERENCES}

1. Torre LA, Bray F, Siegel RL, Ferlay J, Lortet-Tieulent J, Jemal A. Global cancer statistics, 2012. CA Cancer J Clin. 2015;65:87-108.

2. Lozano R, Naghavi M, Foreman K, et al. Global and regional mortality from 235 causes of death for 20 age groups in 1990 and 2010: a systematic analysis for the Global Burden of Disease Study 2010. Lancet. 2012;380:2095-2128.

3. Turkbey B, Brown AM, Sankineni S, Wood BJ, Pinto PA, Choyke PL. Multiparametric prostate magnetic resonance imaging in the evaluation of prostate cancer. CA Cancer J Clin. 2016;66:326-336.

4. De Visschere PJ, Briganti A, Futterer JJ, et al. Role of multiparametric magnetic resonance imaging in early detection of prostate cancer. Insights Imaging. 2016;7:205-214.
5. Heesakkers RA, Hovels AM, Jager GJ, et al. MRI with a lymph-node-specific contrast agent as an alternative to CT scan and lymph-node dissection in patients with prostate cancer: a prospective multicohort study. Lancet Oncol. 2008;9:850-856

6. Hövels AM, Heesakkers RA, Adang EM, et al. The diagnostic accuracy of CT and MRI in the staging of pelvic lymph nodes in patients with prostate cancer: a meta-analysis. Clin Radiol. 2008;63:387-395.

7. Freedland SJ, Presti JC Jr, Amling CL, et al. Time trends in biochemical recurrence after radical prostatectomy: results of the SEARCH database. Urology. 2003;61:736-741.

8. Merseburger AS, Alcaraz A, von Klot CA. Androgen deprivation therapy as backbone therapy in the management of prostate cancer. Onco Targets Ther. 2016;9:7263-7274.

9. de Bono JS, Logothetis CJ, Molina A, et al. Abiraterone and increased survival in metastatic prostate cancer. N Engl J Med. 2011;364:1995-2005.

10. Scher HI, Beer TM, Higano CS, et al. Antitumour activity of MDV3100 in castration-resistant prostate cancer: a phase 1-2 study. Lancet. 2010;375:1437-1446.

11. van Soest RJ, van Royen ME, de Morree ES, et al. Cross-resistance between taxanes and new hormonal agents abiraterone and enzalutamide may affect drug sequence choices in metastatic castration-resistant prostate cancer. Eur $J$ Cancer. 2013;49:3821-3830.

12. Seruga B, Tannock IF. Chemotherapy-based treatment for castration-resistant prostate cancer. J Clin Oncol. 2011;29:3686-3694.

13. de Bono JS, Oudard S, Ozguroglu M, et al. Prednisone plus cabazitaxel or mitoxantrone for metastatic castration-resistant prostate cancer progressing after docetaxel treatment: a randomised open-label trial. Lancet. 2010;376:1147-1154.

14. Fizazi K, Scher HI, Molina A, et al. Abiraterone acetate for treatment of metastatic castration-resistant prostate cancer: final overall survival analysis of the COU-AA-301 randomised, double-blind, placebo-controlled phase 3 study. Lancet Oncol. 2012;13:983-992.

15. Parker C, Nilsson S, Heinrich D, et al. Alpha emitter radium-223 and survival in metastatic prostate cancer. $N$ Engl J Med. 2013;369:213-223.

16. Scher HI, Fizazi K, Saad F, et al. Increased survival with enzalutamide in prostate cancer after chemotherapy. N Engl J Med. 2012;367:1187-1197.

17. Heidenreich A, Bastian PJ, Bellmunt J, et al. EAU guidelines on prostate cancer. Part 1: screening, diagnosis, and local treatment with curative intent-update 2013. Eur Urol. 2014;65:124-137.

18. Schöder H, Herrmann K, Gonen M, et al. 2-[ ${ }^{18}$ F $]$ fluoro-2-deoxyglucose positron emission tomography for the detection of disease in patients with prostatespecific antigen relapse after radical prostatectomy. Clin Cancer Res. 2005;11:4761-4769.

19. Jadvar H. Imaging evaluation of prostate cancer with ${ }^{18} \mathrm{~F}$-fluorodeoxyglucose PET/ CT: utility and limitations. Eur J Nucl Med Mol Imaging. 2013;40(suppl 1):S5-S10.

20. Fanti S, Minozzi S, Castellucci P, et al. PET/CT with ${ }^{11} \mathrm{C}$-choline for evaluation of prostate cancer patients with biochemical recurrence: meta-analysis and critical review of available data. Eur J Nucl Med Mol Imaging. 2016;43:55-69.

21. Evangelista L, Guttilla A, Zattoni F, Muzzio PC, Zattoni F. Utility of choline positron emission tomography/computed tomography for lymph node involvement identification in intermediate- to high-risk prostate cancer: a systematic literature review and meta-analysis. Eur Urol. 2013;63:1040-1048.

22. Nitsch S, Hakenberg OW, Heuschkel M, et al. Evaluation of prostate cancer with ${ }^{11} \mathrm{C}$ - and ${ }^{18} \mathrm{~F}$-choline PET/CT: diagnosis and initial staging. J Nucl Med. 2016;57(suppl):38S-42S.

23. Souvatzoglou M, Weirich $\mathrm{G}$, Schwarzenboeck S, et al. The sensitivity of $\left[{ }^{11} \mathrm{C}\right]$ choline PET/CT to localize prostate cancer depends on the tumor configuration. Clin Cancer Res. 2011;17:3751-3759.

24. Schwarzenböck SM, Eiber M, Kundt G, et al. Prospective evaluation of $\left[{ }^{11} \mathrm{C}\right]$ choline PET/CT in therapy response assessment of standardized docetaxel firstline chemotherapy in patients with advanced castration refractory prostate cancer. Eur J Nucl Med Mol Imaging. 2016;43:2105-2113.

25. Zukotynski KA, Kim CK, Gerbaudo VH, et al. ${ }^{18}$ F-FDG-PET/CT and ${ }^{18} \mathrm{~F}-\mathrm{NaF}-$ $\mathrm{PET} / \mathrm{CT}$ in men with castrate-resistant prostate cancer. Am J Nucl Med Mol Imaging. 2014;5:72-82.

26. Nanni $\mathrm{C}$, Schiavina R, Boschi $\mathrm{S}$, et al. Comparison of ${ }^{18} \mathrm{~F}-\mathrm{FACBC}$ and ${ }^{11} \mathrm{C}$ choline PET/CT in patients with radically treated prostate cancer and biochemical relapse: preliminary results. Eur J Nucl Med Mol Imaging. 2013;40(suppl 1):S11-S17.

27. Ren J, Yuan L, Wen G, Yang J. The value of anti-1-amino-3- ${ }^{18} \mathrm{~F}$-fluorocyclobutane1-carboxylic acid PET/CT in the diagnosis of recurrent prostate carcinoma: a metaanalysis. Acta Radiol. 2016;57:487-493.

28. DeMarzo AM, Nelson WG, Isaacs WB, Epstein JI. Pathological and molecular aspects of prostate cancer. Lancet. 2003;361:955-964.

29. Ghosh A, Heston WDW. Tumor target prostate specific membrane antigen (PSMA) and its regulation in prostate cancer. J Cell Biochem. 2004;91:528-539. 
30. Wright GL, Haley C, Beckett ML, Schellhammer PF. Expression of prostatespecific membrane antigen in normal, benign, and malignant prostate tissues. Urol Oncol. 1995;1:18-28.

31. Huang E, Teh BS, Mody DR, Carpenter LS, Butler EB. Prostate adenocarcinoma presenting with inguinal lymphadenopathy. Urology. 2003;61:463.

32. Silver DA, Pellicer I, Fair WR, Heston WD, Cordon-Cardo C. Prostate-specific membrane antigen expression in normal and malignant human tissues. Clin Cancer Res. 1997;3:81-85.

33. Mannweiler S, Amersdorfer P, Trajanoski S, Terrett JA, King D, Mehes G. Heterogeneity of prostate-specific membrane antigen (PSMA) expression in prostate carcinoma with distant metastasis. Pathol Oncol Res. 2009;15:167-172.

34. Bostwick DG, Pacelli A, Blute M, Roche P, Murphy GP. Prostate specific membrane antigen expression in prostatic intraepithelial neoplasia and adenocarcinoma: a study of 184 cases. Cancer. 1998;82:2256-2261.

35. Troyer JK, Beckett ML, Wright GL Jr. Detection and characterization of the prostate-specific membrane antigen (PSMA) in tissue extracts and body fluids. Int J Cancer: 1995;62:552-558.

36. Budäus L, Leyh-Bannurah SR, Salomon G, et al. Initial experience of ${ }^{68} \mathrm{Ga}-$ PSMA PET/CT imaging in high-risk prostate cancer patients prior to radical prostatectomy. Eur Urol. 2016;69:393-396.

37. Maurer T, Gschwend JE, Rauscher I, et al. Diagnostic efficacy of ${ }^{68}$ galliumPSMA positron emission tomography compared to conventional imaging for lymph node staging of 130 consecutive patients with intermediate to high risk prostate cancer. J Urol. 2016;195:1436-1443.

38. Chang SS. Overview of prostate-specific membrane antigen. Rev Urol. 2004;6 (suppl 10):S13-S18.

39. Rajasekaran SA, Anilkumar G, Oshima E, et al. A novel cytoplasmic tail MXXXL motif mediates the internalization of prostate-specific membrane antigen. Mol Biol Cell. 2003;14:4835-4845.

40. Commandeur LC, Parsons JR. Degradation of halogenated aromatic compounds. Biodegradation. 1990;1:207-220.

41. Hillier SM, Maresca KP, Lu G, et al. ${ }^{99 \mathrm{~m}}$ Tc-labeled small-molecule inhibitors of prostate-specific membrane antigen for molecular imaging of prostate cancer. $J$ Nucl Med. 2013;54:1369-1376.

42. Noss KR, Wolfe SA, Grimes SR. Upregulation of prostate specific membrane antigen/folate hydrolase transcription by an enhancer. Gene. 2002;285:247-256.

43. Evans MJ, Smith-Jones PM, Wongvipat J, et al. Noninvasive measurement of androgen receptor signaling with a positron-emitting radiopharmaceutical that targets prostate-specific membrane antigen. Proc Natl Acad Sci USA. 2011;108:9578-9582.

44. Wright GL Jr, Grob BM, Haley C, et al. Upregulation of prostate-specific membrane antigen after androgen-deprivation therapy. Urology. 1996;48:326334.

45. Murphy G, Ragde H, Kenny G, et al. Comparison of prostate specific membrane antigen, and prostate specific antigen levels in prostatic cancer patients. Anticancer Res. 1995;15:1473-1479.

46. Liu T, Wu LY, Fulton MD, Johnson JM, Berkman CE. Prolonged androgen deprivation leads to downregulation of androgen receptor and prostate-specific membrane antigen in prostate cancer cells. Int J Oncol. 2012;41:2087-2092.

47. Meller B, Bremmer F, Sahlmann CO, et al. Alterations in androgen deprivation enhanced prostate-specific membrane antigen (PSMA) expression in prostate cancer cells as a target for diagnostics and therapy. EJNMMI Res. 2015;5:66.

48. Hope TA, Truillet C, Ehman EC, et al. ${ }^{68} \mathrm{Ga}$-PSMA-11 PET imaging of response to androgen receptor inhibition: first human experience. $J$ Nucl Med. 2017;58:81-84

49. Chang SS, O'Keefe DS, Bacich DJ, Reuter VE, Heston WD, Gaudin PB. Prostate-specific membrane antigen is produced in tumor-associated neovasculature. Clin Cancer Res. 1999;5:2674-2681.

50. Chang SS, Reuter VE, Heston WD, Bander NH, Grauer LS, Gaudin PB. Five different anti-prostate-specific membrane antigen (PSMA) antibodies confirm PSMA expression in tumor-associated neovasculature. Cancer Res. 1999; 59:3192-3198.

51. Chang SS, Reuter VE, Heston WD, Gaudin PB. Metastatic renal cell carcinoma neovasculature expresses prostate-specific membrane antigen. Urology. 2001; 57:801-805.

52. Haffner MC, Kronberger IE, Ross JS, et al. Prostate-specific membrane antigen expression in the neovasculature of gastric and colorectal cancers. Hum Pathol. 2009;40:1754-1761.

53. Samplaski MK, Heston W, Elson P, Magi-Galluzzi C, Hansel DE. Folate hydrolase (prostate-specific membrane antigen) 1 expression in bladder cancer subtypes and associated tumor neovasculature. Mod Pathol. 2011;24:1521-1529.

54. Rauscher I, Maurer T, Fendler WP, Sommer WH, Schwaiger M, Eiber M. ${ }^{68} \mathrm{Ga}-$ PSMA ligand PET/CT in patients with prostate cancer: how we review and report. Cancer Imaging. 2016;16:14.
55. Bander NH. Technology insight: monoclonal antibody imaging of prostate cancer. Nat Clin Pract Urol. 2006;3:216-225.

56. Mesters JR, Barinka C, Li W, et al. Structure of glutamate carboxypeptidase II, a drug target in neuronal damage and prostate cancer. EMBO J. 2006;25:13751384.

57. Beheshti M, Kunit T, Haim S, et al. BAY 1075553 PET-CT for staging and restaging prostate cancer patients: comparison with $\left[{ }^{18} \mathrm{~F}\right]$ fluorocholine PET-CT (phase I study). Mol Imaging Biol. 2015;17:424-433.

58. Yang X, Mease RC, Pullambhatla M, et al. $\left[{ }^{18} \mathrm{~F}\right]$ fluorobenzoyllysinepentanedioic acid carbamates: new scaffolds for positron emission tomography (PET) imaging of prostate-specific membrane antigen (PSMA). J Med Chem. 2016;59:206-218.

59. Mease RC, Foss CA, Pomper MG. PET imaging in prostate cancer: focus on prostate-specific membrane antigen. Curr Top Med Chem. 2013;13:951-962.

60. Lütje S, Heskamp S, Cornelissen AS, et al. PSMA ligands for radionuclide imaging and therapy of prostate cancer: clinical status. Theranostics. 2015; 5:1388-1401

61. Rowe SP, Drzezga A, Neumaier B, et al. Prostate-specific membrane antigentargeted radiohalogenated PET and therapeutic agents for prostate cancer. $J$ Nucl Med. 2016;57(suppl):90S-96S.

62. Barrett JA, Coleman RE, Goldsmith SJ, et al. First-in-man evaluation of 2 highaffinity PSMA-avid small molecules for imaging prostate cancer. $\mathrm{J} \mathrm{Nucl} \mathrm{Med.}$ 2013;54:380-387.

63. Cho SY, Gage KL, Mease RC, et al. Biodistribution, tumor detection, and radiation dosimetry of ${ }^{18} \mathrm{~F}$-DCFBC, a low-molecular-weight inhibitor of prostate-specific membrane antigen, in patients with metastatic prostate cancer. J Nucl Med. 2012;53:1883-1891.

64. Eder M, Neels O, Müller M, et al. Novel preclinical and radiopharmaceutical aspects of $\left[{ }^{68} \mathrm{Ga}\right] \mathrm{Ga}-P S M A-H B E D-C C$ : a new PET tracer for imaging of prostate cancer. Pharmaceuticals (Basel). 2014;7:779-796.

65. Zechmann CM, Afshar-Oromieh A, Armor T, et al. Radiation dosimetry and first therapy results with a ${ }^{124} \mathrm{I} /{ }^{131} \mathrm{I}$-labeled small molecule (MIP-1095) targeting PSMA for prostate cancer therapy. Eur J Nucl Med Mol Imaging. 2014;41:1280-1292.

66. Afshar-Oromieh A, Hetzheim H, Kratochwil C, et al. The theranostic PSMA ligand PSMA-617 in the diagnosis of prostate cancer by PET/CT: biodistribution in humans, radiation dosimetry, and first evaluation of tumor lesions. $J \mathrm{Nucl}$ Med. 2015;56:1697-1705.

67. Weineisen M, Schottelius M, Simecek J, et al. ${ }^{68} \mathrm{Ga}$ - and ${ }^{177}$ Lu-labeled PSMA I\&T: optimization of a PSMA-targeted theranostic concept and first proof-ofconcept human studies. J Nucl Med. 2015;56:1169-1176.

68. Chen Y, Pullambhatla M, Foss CA, et al. 2-(3-\{1-Carboxy-5-[(6- $\left[{ }^{18}\right.$ F $]$ fluoropyridine-3-carbonyl)-amino]-pentyl $\}$-ureido)-pentanedioic acid, $\left[{ }^{18} \mathrm{~F}\right] \mathrm{DCFPyL}$, a PSMA-based PET imaging agent for prostate cancer. Clin Cancer Res. 2011;17:7645-7653.

69. Giesel FL, Hadaschik B, Cardinale J, et al. F-18 labelled PSMA-1007: biodistribution, radiation dosimetry and histopathological validation of tumor lesions in prostate cancer patients. Eur J Nucl Med Mol Imaging. 2017;44:678-688.

70. Sanchez-Crespo A. Comparison of gallium-68 and fluorine-18 imaging characteristics in positron emission tomography. Appl Radiat Isot. 2013;76:55-62.

71. Robu S, Schottelius M, Eiber M, et al. Preclinical evaluation and first patient application of ${ }^{99 \mathrm{~m}} \mathrm{Tc}-\mathrm{PSMA}-\mathrm{I} \& \mathrm{~S}$ for SPECT imaging and radioguided surgery in prostate cancer. $J$ Nucl Med. 2017;58:235-242.

72. Afshar-Oromieh A, Haberkorn U, Eder M, Eisenhut M, Zechmann CM. $\left[{ }^{68} \mathrm{Ga}\right]$ gallium-labelled PSMA ligand as superior PET tracer for the diagnosis of prostate cancer: comparison with ${ }^{18} \mathrm{~F}-\mathrm{FECH}$. Eur J Nucl Med Mol Imaging. 2012;39:1085-1086.

73. Perera M, Papa N, Christidis D, et al. Sensitivity, specificity, and predictors of positive ${ }^{68} \mathrm{Ga}$-prostate-specific membrane antigen positron emission tomography in advanced prostate cancer: a systematic review and meta-analysis. Eur Urol. 2016;70:926-937.

74. Afshar-Oromieh A, Avtzi E, Giesel FL, et al. The diagnostic value of PET/CT imaging with the ${ }^{68} \mathrm{Ga}$-labelled PSMA ligand HBED-CC in the diagnosis of recurrent prostate cancer. Eur J Nucl Med Mol Imaging. 2015;42:197-209.

75. Eiber M, Maurer T, Souvatzoglou M, et al. Evaluation of hybrid ${ }^{68} \mathrm{Ga}-\mathrm{PSMA}$ ligand PET/CT in 248 patients with biochemical recurrence after radical prostatectomy. J Nucl Med. 2015;56:668-674.

76. Ceci F, Uprimny C, Nilica B, et al. ${ }^{68} \mathrm{Ga}$-PSMA PET/CT for restaging recurrent prostate cancer: which factors are associated with PET/CT detection rate? Eur J Nucl Med Mol Imaging. 2015;42:1284-1294.

77. Berliner C, Tienken M, Frenzel T, et al. Detection rate of PET/CT in patients with biochemical relapse of prostate cancer using $\left[{ }^{68} \mathrm{Ga}\right]$ PSMA I\&T and comparison with published data of $\left[{ }^{68} \mathrm{Ga}\right]$ PSMA HBED-CC. Eur J Nucl Med Mol Imaging. 2017;44:670-677. 
78. Morigi JJ, Stricker PD, van Leeuwen PJ, et al. Prospective comparison of ${ }^{18} \mathrm{~F}-$ fluoromethylcholine versus ${ }^{68} \mathrm{Ga}$-PSMA PET/CT in prostate cancer patients who have rising PSA after curative treatment and are being considered for targeted therapy. J Nucl Med. 2015;56:1185-1190.

79. Schwenck J, Rempp H, Reischl G, et al. Comparison of ${ }^{68} \mathrm{Ga}$-labelled PSMA11 and ${ }^{11} \mathrm{C}$-choline in the detection of prostate cancer metastases by PET/CT. Eur J Nucl Med Mol Imaging. 2017;44:92-101.

80. Afshar-Oromieh A, Zechmann CM, Malcher A, et al. Comparison of PET imaging with a ${ }^{68} \mathrm{Ga}$-labelled PSMA ligand and ${ }^{18} \mathrm{~F}$-choline-based PET/CT for the diagnosis of recurrent prostate cancer. Eur J Nucl Med Mol Imaging. 2014;41:11-20.

81. van Leeuwen PJ, Emmett L, Ho B, et al. Prospective evaluation of ${ }^{68}$ galliumprostate-specific membrane antigen positron emission tomography/computerized tomography for preoperative lymph node staging in prostate cancer. $B J U$ Int. 2017;119:209-215.

82. Rowe SP, Macura KJ, Ciarallo A, et al. Comparison of prostate-specific membrane antigen-based ${ }^{18} \mathrm{~F}$-DCFBC PET/CT to conventional imaging modalities for detection of hormone-naive and castration-resistant metastatic prostate cancer. J Nucl Med. 2016;57:46-53.

83. Szabo Z, Mena E, Rowe SP, et al. Initial evaluation of $\left[{ }^{18} \mathrm{~F}\right] \mathrm{DCFPyL}$ for prostate-specific membrane antigen (PSMA)-targeted PET imaging of prostate cancer. Mol Imaging Biol. 2015;17:565-574.

84. Rowe SP, Macura KJ, Mena E, et al. PSMA-based [ $\left.{ }^{18} \mathrm{~F}\right] \mathrm{DCFPyL}$ PET/CT is superior to conventional imaging for lesion detection in patients with metastatic prostate cancer. Mol Imaging Biol. 2016;18:411-419.

85. Dietlein M, Kobe C, Kuhnert G, et al. Comparison of $\left[{ }^{18} \mathrm{~F}\right] \mathrm{DCFPyL}$ and $\left[{ }^{68} \mathrm{Ga}\right]$ Ga-PSMA-HBED-CC for PSMA-PET imaging in patients with relapsed prostate cancer. Mol Imaging Biol. 2015;17:575-584.

86. Dietlein F, Kobe C, Neubauer S, et al. PSA-stratified performance of ${ }^{18} \mathrm{~F}$ - and ${ }^{68} \mathrm{Ga}$-labeled tracers in PSMA-PET imaging of patients with biochemical recurrence of prostate cancer. $J$ Nucl Med. December 1, 2016 [Epub ahead of print].

87. Giesel FL, Kesch C, Yun M, et al. ${ }^{18}$ F-PSMA-1007 PET/CT detects micrometastases in a patient with biochemically recurrent prostate cancer. Clin Genitourin Cancer. December 29, 2016 [Epub ahead of print].

88. Vallabhajosula S, Nikolopoulou A, Babich JW, et al. ${ }^{99 \mathrm{~m}} \mathrm{Tc}$-labeled smallmolecule inhibitors of prostate-specific membrane antigen: pharmacokinetics and biodistribution studies in healthy subjects and patients with metastatic prostate cancer. J Nucl Med. 2014;55:1791-1798.

89. Reinfelder J, Kuwert T, Beck M, et al. First experience with SPECT/CT using a ${ }^{99 \mathrm{~m}} \mathrm{Tc}$-labeled inhibitor for prostate-specific membrane antigen in patients with biochemical recurrence of prostate cancer. Clin Nucl Med. 2017;42:26-33

90. Rauscher I, Maurer T, Souvatzoglou M, et al. Intrapatient comparison of ${ }^{111} \mathrm{In}$ PSMA I\&T SPECT/CT and hybrid ${ }^{68} \mathrm{Ga}-\mathrm{HBED}-\mathrm{CC}$ PSMA PET in patients with early recurrent prostate cancer. Clin Nucl Med. 2016;41:e397-e402.

91. Sterzing F, Kratochwil C, Fiedler H, et al. ${ }^{68}$ Ga-PSMA-11 PET/CT: a new technique with high potential for the radiotherapeutic management of prostate cancer patients. Eur J Nucl Med Mol Imaging. 2016;43:34-41.

92. Dewes S, Schiller K, Sauter K, et al. Integration of ${ }^{68}$ Ga-PSMA-PET imaging in planning of primary definitive radiotherapy in prostate cancer: a retrospective study. Radiat Oncol. 2016;11:73.

93. Bräuer A, Rahbar K, Konnert J, Bögemann M, Stegger L. Diagnostic value of additional ${ }^{68} \mathrm{Ga}$-PSMA-PET before ${ }^{223} \mathrm{Ra}$-dichloride therapy in patients with metastatic prostate carcinoma. Nuklearmedizin. 2017;56:14-22.

94. Herlemann A, Wenter V, Kretschmer A, et al. ${ }^{68} \mathrm{Ga}$-PSMA positron emission tomography/computed tomography provides accurate staging of lymph node regions prior to lymph node dissection in patients with prostate cancer. Eur Urol. 2016;70:553-557.

95. Hijazi S, Meller B, Leitsmann C, et al. Pelvic lymph node dissection for nodal oligometastatic prostate cancer detected by ${ }^{68} \mathrm{Ga}-\mathrm{PSMA}$-positron emission tomography/computerized tomography. Prostate. 2015;75:1934-1940.

96. Pyka T, Okamoto S, Dahlbender M, et al. Comparison of bone scintigraphy and ${ }^{68} \mathrm{Ga}$-PSMA PET for skeletal staging in prostate cancer. Eur J Nucl Med Mol Imaging. 2016;43:2114-2121.

97. Fendler WP, Schmidt DF, Wenter V, et al. ${ }^{68}$ Ga-PSMA PET/CT detects the location and extent of primary prostate cancer. J Nucl Med. 2016;57:1720-1725.

98. Rahbar K, Weckesser M, Huss S, et al. Correlation of intraprostatic tumor extent with ${ }^{68} \mathrm{Ga}$-PSMA distribution in patients with prostate cancer. $\mathrm{J} \mathrm{Nucl}$ Med. 2016;57:563-567.

99. Eiber M, Weirich G, Holzapfel K, et al. Simultaneous ${ }^{68}$ Ga-PSMA HBED-CC PET/MRI improves the localization of primary prostate cancer. Eur Urol. 2016;70:829-836.

100. Zamboglou C, Drendel V, Jilg CA, et al. Comparison of ${ }^{68} \mathrm{Ga}-\mathrm{HBED}-\mathrm{CC}$ PSMA-PET/CT and multiparametric MRI for gross tumour volume detection in patients with primary prostate cancer based on slice by slice comparison with histopathology. Theranostics. 2017;7:228-237.
101. Zamboglou C, Schiller F, Fechter T, et al. ${ }^{68}$ Ga-HBED-CC-PSMA PET/CT versus histopathology in primary localized prostate cancer: a voxel-wise comparison. Theranostics. 2016;6:1619-1628.

102. Giesel FL, Sterzing F, Schlemmer HP, et al. Intra-individual comparison of ${ }^{68} \mathrm{Ga}$-PSMA-11-PET/CT and multi-parametric MR for imaging of primary prostate cancer. Eur J Nucl Med Mol Imaging. 2016;43:1400-1406.

103. Rowe SP, Gage KL, Faraj SF, et al. ${ }^{18}$ F-DCFBC PET/CT for PSMA-based detection and characterization of primary prostate cancer. J Nucl Med. 2015; 56:1003-1010.

104. Yu JB, Makarov DV, Gross C. A new formula for prostate cancer lymph node risk. Int J Radiat Oncol Biol Phys. 2011;80:69-75.

105. Roach M III, Hanks G, Thames H Jr., et al. Defining biochemical failure following radiotherapy with or without hormonal therapy in men with clinically localized prostate cancer: recommendations of the RTOG-ASTRO Phoenix Consensus Conference. Int J Radiat Oncol Biol Phys. 2006;65:965-974.

106. Zamboglou C, Wieser G, Hennies S, et al. MRI versus ${ }^{68} \mathrm{Ga}$-PSMA PET/CT for gross tumour volume delineation in radiation treatment planning of primary prostate cancer. Eur J Nucl Med Mol Imaging. 2016;43:889-897.

107. Tagawa ST, Milowsky MI, Morris M, et al. Phase II study of lutetium-177labeled anti-prostate-specific membrane antigen monoclonal antibody J591 for metastatic castration-resistant prostate cancer. Clin Cancer Res. 2013;19:51825191.

108. Vallabhajosula S, Nikolopoulou A, Jhanwar YS, et al. Radioimmunotherapy of metastatic prostate cancer with ${ }^{177} \mathrm{Lu}$-DOTAhuJ591 anti prostate specific membrane antigen specific monoclonal antibody. Curr Radiopharm. 2016;9:44-53.

109. Kassis AI. Therapeutic radionuclides: biophysical and radiobiologic principles. Semin Nucl Med. 2008;38:358-366.

110. Rahbar K, Ahmadzadehfar H, Kratochwil C, et al. German multicenter study investigating ${ }^{177} \mathrm{Lu}-\mathrm{PSMA}-617$ radioligand therapy in advanced prostate cancer patients. J Nucl Med. 2017;58:85-90.

111. Kratochwil C, Giesel FL, Eder M, et al. $\left[{ }^{177}\right.$ Lu]lutetium-labelled PSMA ligandinduced remission in a patient with metastatic prostate cancer. Eur J Nucl Med Mol Imaging. 2015;42:987-988.

112. Baum RP, Kulkarni HR, Schuchardt C, et al. ${ }^{177} \mathrm{Lu}$-labeled prostate-specific membrane antigen radioligand therapy of metastatic castration-resistant prostate cancer: safety and efficacy. J Nucl Med. 2016;57:1006-1013.

113. Rahbar K, Schmidt M, Heinzel A, et al. Response and tolerability of a single dose of ${ }^{177} \mathrm{Lu}$-PSMA-617 in patients with metastatic castration-resistant prostate cancer: a multicenter retrospective analysis. J Nucl Med. 2016;57:1334-1338.

114. Ahmadzadehfar H, Rahbar K, Kürpig S, et al. Early side effects and first results of radioligand therapy with ${ }^{177} \mathrm{Lu}-\mathrm{DKFZ}-617$ PSMA of castrate-resistant metastatic prostate cancer: a two-centre study. EJNMMI Res. 2015;5:114.

115. Fendler WP, Reinhardt S, Ilhan H, et al. Preliminary experience with dosimetry, response and patient reported outcome after ${ }^{177} \mathrm{Lu}-\mathrm{PSMA}-617$ therapy for metastatic castration-resistant prostate cancer. Oncotarget. 2017;8:3581-3590.

116. Heck MM, Retz M, D'Alessandria C, et al. Systemic radioligand therapy with ${ }^{177} \mathrm{Lu}$ labeled prostate specific membrane antigen ligand for imaging and therapy in patients with metastatic castration resistant prostate cancer. $J$ Urol. 2016;196:382-391.

117. Delker A, Fendler WP, Kratochwil C, et al. Dosimetry for ${ }^{177}$ Lu-DKFZ-PSMA617: a new radiopharmaceutical for the treatment of metastatic prostate cancer. Eur J Nucl Med Mol Imaging. 2016;43:42-51.

118. Kratochwil C, Giesel FL, Stefanova M, et al. PSMA-targeted radionuclide therapy of metastatic castration-resistant prostate cancer with ${ }^{177} \mathrm{Lu}$-labeled PSMA-617. J Nucl Med. 2016;57:1170-1176.

119. Okamoto S, Thieme A, Allmann J, et al. Radiation dosimetry for ${ }^{177}$ Lu-PSMA I\&T in metastatic castration-resistant prostate cancer: absorbed dose in normal organs and tumor lesions. J Nucl Med. 2017;58:445-450.

120. Hohberg M, Eschner W, Schmidt M, et al. Lacrimal glands may represent organs at risk for radionuclide therapy of prostate cancer with $\left[{ }^{177} \mathrm{Lu}\right] \mathrm{DKFZ}$ PSMA-617. Mol Imaging Biol. 2016;18:437-445.

121. Kratochwil C, Bruchertseifer F, Giesel FL, et al. ${ }^{225}$ Ac-PSMA-617 for PSMAtargeted $\alpha$-radiation therapy of metastatic castration-resistant prostate cancer. $J$ Nucl Med. 2016;57:1941-1944.

122. Maurer T, Weirich G, Schottelius M, et al. Prostate-specific membrane antigenradioguided surgery for metastatic lymph nodes in prostate cancer. Eur Urol. 2015;68:530-534.

123. Rauscher I, Düwel C, Wirtz M, et al. Value of ${ }^{111}$ In-prostate-specific membrane antigen (PSMA)-radioguided surgery for salvage lymphadenectomy in recurrent prostate cancer: correlation with histopathology and clinical follow-up. BJU Int. November 10, 2016 [Epub ahead of print].

124. Neuman BP, Eifler JB, Castanares M, et al. Real-time, near-infrared fluorescence imaging with an optimized dye/light source/camera combination for surgical guidance of prostate cancer. Clin Cancer Res. 2015;21:771-780. 